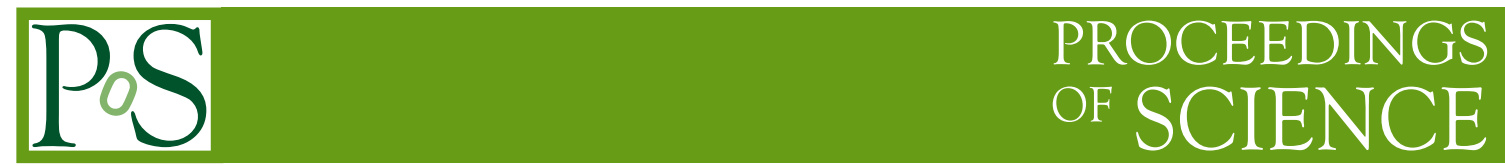

\title{
Symmetries in Large Scale Structure
}

\author{
Lam Hui* \\ Columbia University \\ E-mail: Ih399@columbia.edu
}

We will discuss the role of symmetries in three different areas of large scale structure:

1. how to test the equivalence principle using black holes in centers of galaxies;

2. how to measure gravitational redshifts using parity-violating signatures in correlation functions;

3. how spontaneously broken symmetries give us fully non-perturbative relations between $N$ and $N+1$ point functions.

Frontiers of Fundamental Physics 14 - FFP14,

15-18 July 2014

Aix Marseille University (AMU) Saint-Charles Campus, Marseille

${ }^{*}$ Speaker. 